\title{
Modeling Nondestructive Defect Detection in Additively Manufactured Metallic Structures for Nuclear Applications
}

Nuclear Science and Engineering Division 


\title{
About Argonne National Laboratory
}

Argonne is a U.S. Department of Energy laboratory managed by UChicago Argonne, LLC under contract DE-AC02-06CH11357. The Laboratory's main facility is outside

Chicago, at 9700 South Cass Avenue, Argonne, Illinois 60439. For information about Argonne

and its pioneering science and technology programs, see www.anl.gov.

\section{DOCUMENT AVAILABILITY}

Online Access: U.S. Department of Energy (DOE) reports produced after 1991 and a growing number of pre-1991 documents are available free at OSTI.GOV (http://www.osti.gov/), a service of the US Dept. of Energy's Office of Scientific and Technical Information.

\author{
Reports not in digital format may be purchased by the public from \\ the National Technical Information Service (NTIS): \\ U.S. Department of Commerce \\ National Technical Information \\ Service 5301 Shawnee Rd \\ Alexandria, VA 22312 \\ www.ntis.gov \\ Phone: (800) 553-NTIS (6847) or (703) 605-6000 \\ Fax: (703) 605-6900 \\ Email:orders@ntis.gov
}

\author{
Reports not in digital format are available to DOE and DOE contractors from \\ the Office of Scientific and Technical Information (OSTI): \\ U.S. Department of Energy \\ Office of Scientific and Technical Information \\ P.O. Box 62 \\ Oak Ridge, TN 37831-0062 \\ www.osti.gov \\ Phone: (865) 576-8401 \\ Fax: (865) 576-5728 \\ Email: reports@osti.gov
}

\section{Disclaimer}

This report was prepared as an account of work sponsored by an agency of the United States Government. Neither the United States Government nor any agency thereof, nor UChicago Argonne, LLC, nor any of their employees or officers, makes any warranty, express or implied, or assumes any legal liability or responsibility for the accuracy, completeness, or usefulness of any information, apparatus, product, or process disclosed, or represents that its use would not infringe privately owned rights. Reference herein to any specific commercial product, process, or service by trade name, trademark, manufacturer, or otherwise, does not necessarily constitute or imply its endorsement, recommendation, or favoring by the United States Government or any agency thereof. The views and opinions of document authors expressed herein do not necessarily state or reflect those of the United States Government or any agency thereof, Argonne National Laboratory, or UChicago Argonne, LLC. 


\section{Modeling Nondestructive Defect Detection in Additively Manufactured Metallic Structures for Nuclear Applications}

prepared by

Zoe Fisher ${ }^{1,2}$, Dmitry Shribak ${ }^{1}$, Victoria Ankel ${ }^{1,3}$, Alexander Heifetz ${ }^{1}$,

${ }^{1}$ Nuclear Science and Engineering Division, Argonne National Laboratory

${ }^{2}$ Department of Nuclear Science and Engineering, Massachusetts Institute of Technology

${ }^{3}$ Department of Physics, University of Chicago

October 1, 2020 


\begin{abstract}
The future of quickly, economically produced metallic nuclear reactor parts with minimal supplychain dependence lies in Laser Powder Bed Fusion (LPBF) Additive Manufacturing (AM): a 3D printing method involving laser melting and net shaping stainless steel and Inconel metallic powder into a solid structure. However, intrinsic features in LPBF frequently leads to the formation of materials defects, such as pores, within 3D printed structures. As safe long-term use in energy applications requires knowledge of all relevant defects before deployment in a reactor, we must develop methods for nondestructive detection of these defects. We are investigating Pulsed Thermal Tomography (PTT), which is a non-contact nondestructive imaging method scalable to arbitrary structure size. Thermal tomography (TT) is a computational method for 3D spatial reconstruction of material thermal effusivity from flash or pulsed thermography temperature data cube. Thermography data cube consists of $2 \mathrm{D}$ surface temperature measurements at different times. The objective of the present work is to investigate limits on defect detection in AM metallic structures with PTT. To this effect, we modeled PTT with COMSOL heat transfer computer simulations. We developed a layered media COMSOL simulation consisting of a Stainless Steel 316 (SS316) plate with an internal layer of un-sintered SS316 powder. Thermophysical properties of the powder layer were modeled with equivalent volume mixing model. To account for partial sintering at the boundary of the defect, the transition between solid and powder layers was modeled as a Gaussian. Using data from COMSOL simulations, we reconstructed depth-dependent thermal effusivity, which allowed defect visibility estimation. A series of parametric studies determined that at $1 \mathrm{~mm}$ depth, $50 \mu \mathrm{m}$ is the smallest detectable defect. In addition, classification of the defects which can lead to early fatigue of the metallic structure in a reactor is briefly discussed.
\end{abstract}




\section{Introduction}

Additive manufacturing ( $\mathrm{AM}$ ) or 3D printing enjoys broad-reaching relevance, its usefulness untethered to a single field of science. Most recently, metallic 3D printing in the form of Laser Powder Bed Fusion (LPBF) has emerged as a promising cost-efficient and rapid manufacturing method for new reactor construction, as well as for replacement of aging parts in existing reactors. In AM, the structure stereolithography file is uploaded onto the $3 \mathrm{D}$ printer computer. As depicted in Figure 1, LPBF process consists of building a metallic structure from powder layer by layer [1]. To form a new layer, microscopic metallic powder is poured onto the "bed" and flattened with a roller. The powder is spatially selectively melted with a high-power laser. Computer controlled mirrors steer the laser beam follow the pattern in the stereolithography file. Upon solidification, melt pools fuse with the solid structure to form a new structure layer.
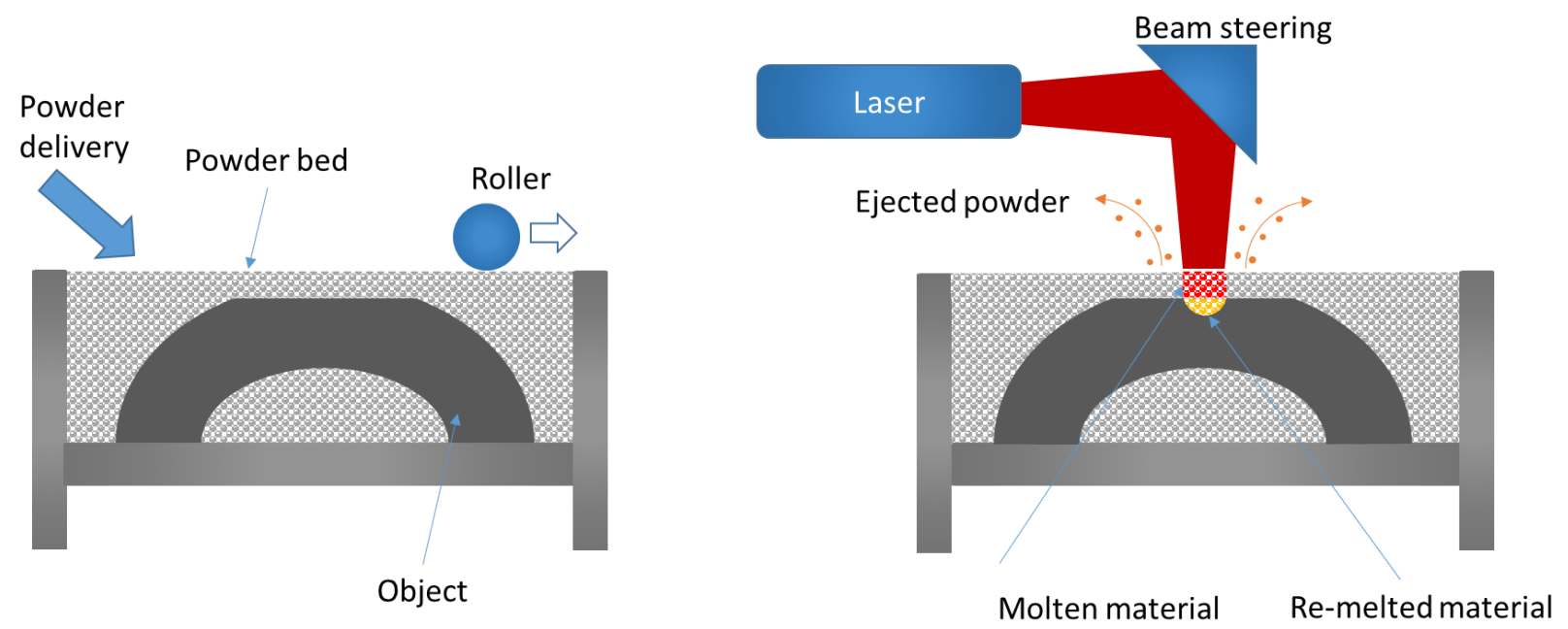

Figure 1 - Schematics of LPBF process [1].

Because of intrinsic features of the LPBF process, such as partial melting of powder grains or improper overlap of melt pools, 3D printed metallic structure may contain pores [2,3]. Three main types of pores occurring during AM process are gas, keyholes and lack of fusion [4]. Keyhole pores, which are caused by an excess of input energy during the melting process, typically have spheroidal shapes and dimensions on the order of tens to hundreds of microns. Lack of fusion pores, which are caused by a lack of input energy to the powder bed during the melting process, have irregular shapes and dimensions on the order of tens to hundreds of microns. Gas pores, which are related to gas trapped in metal during melting and solidification, have spherical shapes and 
dimensions on the order of microns. When subjected to thermal stresses in a nuclear reactor, microscopic defects have the potential to cause premature structure failure.

Because of low reproducibility of AM process, each AM structure needs to be examined nondestructively before deployment in a nuclear reactor. In principle, high resolution imaging of internal density defects can be obtained with X-ray computed tomography (CT). However, this method is applicable only to small structures shaped in the form of symmetrical body of revolution (spheres or cylinders). Nondestructive imaging of the actual AM structure with complex geometry is highly non-trivial. Methods such as ultrasonic tomography require direct contact of the structure with the scanning probe. This has disadvantages since AM structures frequently have a rough finish, and imaging with a scanning probe takes up copious amounts of time. Pulsed Thermal Tomography (PTT) offers potentially promising approach which is non-contact, and images are formed in one measurement with a megapixel camera $[1,5,6]$. The objective of this study is to estimate limits of internal density defect detection in a metallic structure with PTT using computer simulations. 


\section{Pulsed Thermal Tomography Algorithm}

PTT is a computational method for reconstructing 3D thermal effusivity from flash thermography measurements. Thermal effusivity is a measure of how a material exchanges heat with its surroundings. When reconstructed with PPT, effusivity fluctuations can reveal presence of internal defects visibility and the means by which we judge defects. Figure 2 shows a schematic description of PTT [5]. In the imaging setup a flash lamp deposits a thermal pulse on a sample, which in turn expels a temperature signal that an infrared (IR) camera picks up and records. The IR camera stores the real-time surface temperature as a data cube, $T(x, y, t)$.

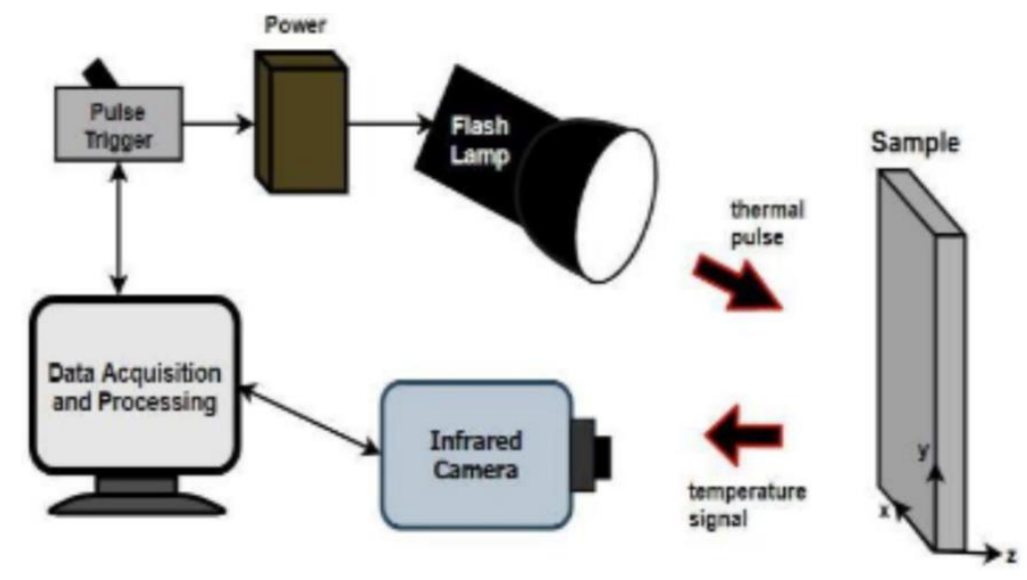

Figure 2 - PTT schematic diagram [5].

The reconstruction algorithm obtains thermal effusivity $e(x, y, z)$ from the time-dependent surface temperature $T(x, y, t)$ measurements [6]. The reconstruciton model assumes onedimensional heat propagation along the $z$-coordinate, which represents depth. The transverse plane contains the $x$ and $y$ coordinates, making 1D heat diffusion

$$
\frac{\partial T}{\partial t}=\alpha \frac{\partial^{2} T}{\partial z^{2}} .
$$

Thermal diffusivity $\alpha$ is dependent on thermal conductivity $k$, density $\rho$, and specific heat $c$ as

$$
\alpha=k / \rho c .
$$

Temperature governs each of these variables. These variables also define thermal effusivity as

$$
e=\sqrt{\rho c k} .
$$


When the flash lamp deposits thermal energy onto the structure surface (instantaneously in theory), the heat diffuses into the material bulk. The thermal wavefront depth's in the material $z$ as a function of time $t$ since the time of heat deposition is

$$
z=\sqrt{\pi \alpha t} .
$$

Applying Equation (1) to semi-infinite slabs produces effusivity as a function of time $e(t) . e(t)$, when united with Equation (4), becomes effusivity as a function of depth $e(z)[5,6]$.

$$
e(z)=\left.z \frac{2 Q}{\pi \sqrt{\alpha}} \frac{d}{d t}\left(\frac{1}{T(t)}\right)\right|_{t=z^{2} / \pi \alpha},
$$

where $Q$ is the instantaneously deposited surface thermal energy density. Equation (5) shows that the depth $z$ and the time derivative of inversed surface temperature (when corresponding to depth $z$ ) produce spatial effusivity $e(z)$. Thus, a linear transformation of matrix $T(x, y, t)$ produces the matrix $e(x, y, z)$.

Information about the material internal structure is contained in the surface temperature transient $T(x, y, t)$ and unlocked through the thermal effusivity $e(x, y, z)$. Effusivity functions as such a reliable guideline because the thermal resistance within the plate (especially when ununiform) affects the local surface temperature decay rate. Pores have a lower thermal diffusivity when compared to a solid material, resulting in slower surface temperature decay above regions containing defects. This phenomenon, observable in "hot spots," functions as a tell-tale sign of internal irregularity. All data regarding effusivity is relative, so each result is normalized for relative comparison. 


\section{Model Setup for Computer Simulations}

This study performed in this project involved COMSOL Multiphysics software simulation of SS316 plate with internal low density defect. In our prior work, we investigated detection of internal defect represented by a flat bottom hole (FBH) model [1]. In addition, previously developed COMSOL computational model suffered from numerical noise, which made it difficult to make accurate assessment of minimal detected defect size. In this work, we have developed a COMSOL model based on approach in which a structure was created as a body of revolution. Thus the entire modeled structure was in the shape of disc. Compared to previously used model of a 3D structure, the body of revolution approach resulted in significantly smaller numerical noise and faster computations.

A layered media model was constructed, in which the total thickness of the plate $(5 \mathrm{~mm})$ was much smaller than the radius of the plate $(30 \mathrm{~cm})$. A defect was represented by an internal low density layer parallel the disc surface, which extended through the entire disc. The lowest density defect considered was represented by un-sintered metallic powder. Such defect would be created if the 3D metal printer laser fails to melt an entire pocket of powder. Higher density defects considered in this study represented partially fused powders. Unlike solid SS316 phase, metallic SS316 powder is a more difficult phase to model. The random close packing (RCP) model accurately represents the metallic powder. According to RCP, monodispersed spheres poured onto a bed have a minimum void fraction of $\varepsilon=0.35$ and corresponding maximum packing density of $\eta=0.65$ [7]. Using the values of solid SS316 density $\rho$ and heat capacity $c$ [8], we calculated those of powder SS316 as linearly scaled quantities (subscript $p$ included for differentiation of powder from solid):

$$
\rho_{p}=\eta \rho, c_{p}=\eta c, k_{p}=\eta k
$$

It should be noted that the relationship between thermal conductivity $k$ in powder and solid SS316 as function of packing fraction is more complicated than that of the density and heat capacity [9]. However, in this study we parameterized the thermal conductivity $k$ for defects with packing fractions. After initial investigation with sharp transitions between solid and powder layers, we concluded that using a Gaussian curve to transition from the solid phase to the powder phase defect results on lowest numerical noise. Effusivity of the structure with defect modeled as subtracted 
Gaussian is shown in Figure 3. The entire structure is $5 \mathrm{~mm}$ thick, with the defect located $1 \mathrm{~mm}$ beneath the surface and infinite in the transverse $x y$ plane.

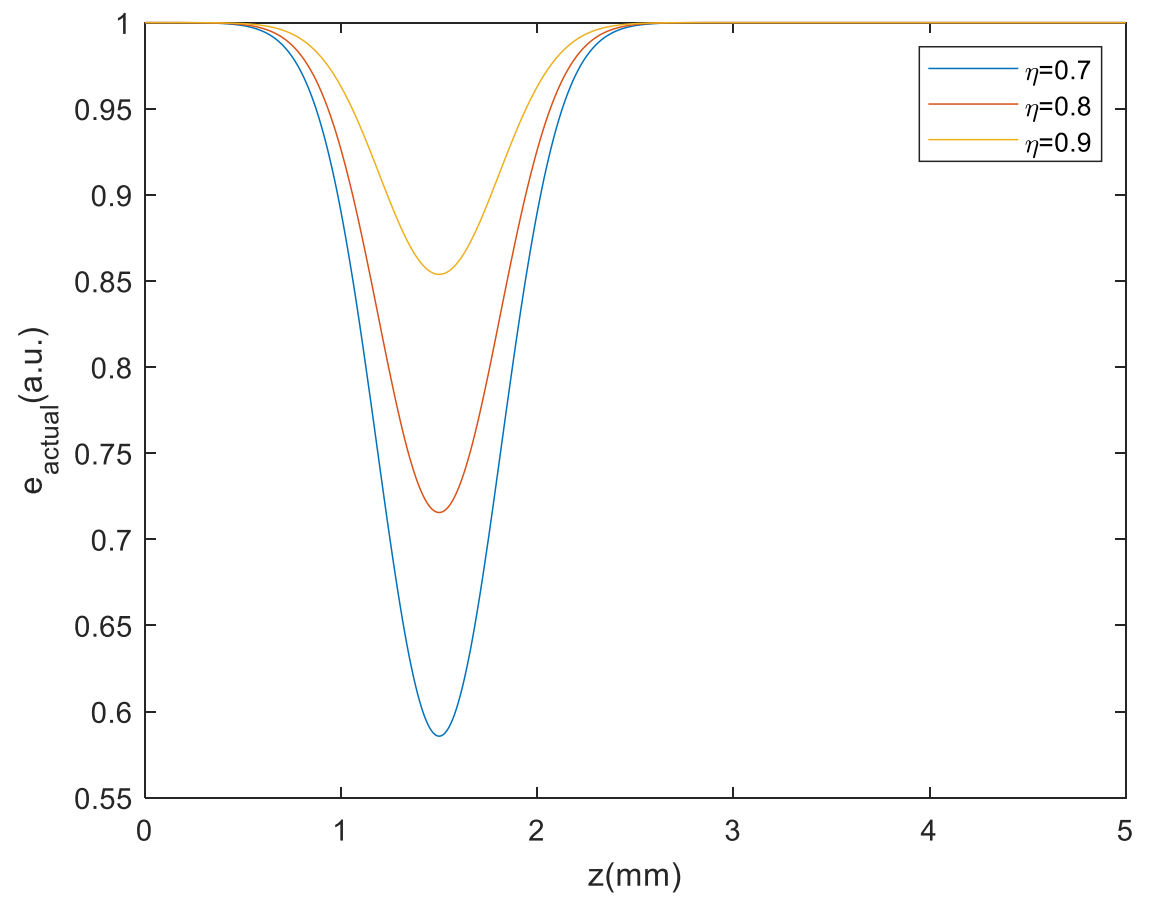

Figure 3 - Effusivity profile of the 5mm-thick SS316 structure with Gaussian curves representing different packing fractions for a $1 \mathrm{~mm}$-thick defect located at $1 \mathrm{~mm}$ depth. 


\section{Results of Computer Simulations of Defect Detection with PTT}

We conducted a parametric study to investigate the visibility of defect modeled as powder layers for the range of packing fractions $\eta=0.6$ to $\eta=1$. As expected, the largest visibility, or change in effusivity (calculated by subtracting the solid plate's effusivity at a certain depth $e(z)$ by the defect under investigation at the same depth $z$ ), is found in the structure with lowest packing with $\eta=0.6$. Figure 4 illustrates the results of computer simulations.

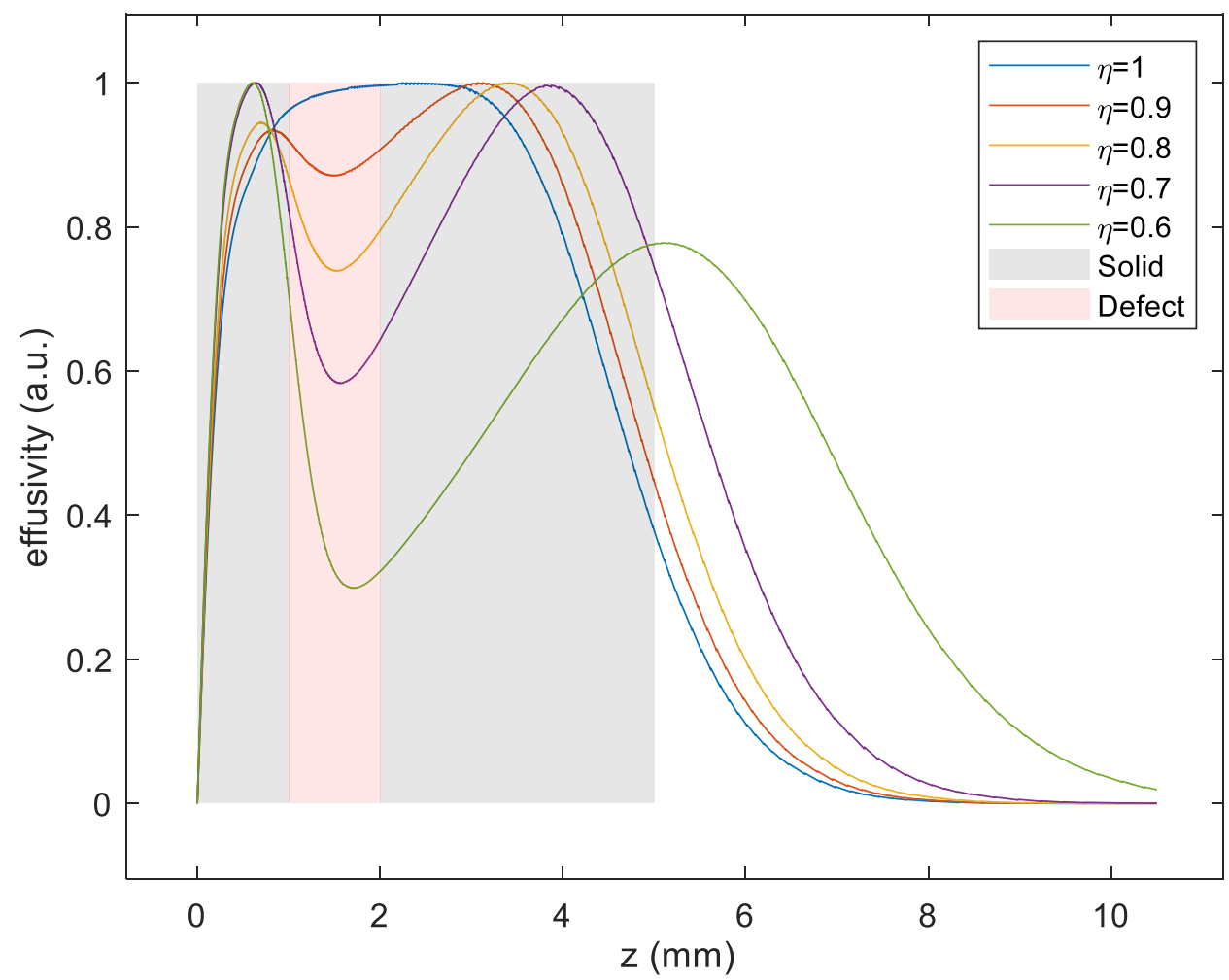

Figure 4 - Reconstructed effusivity as a function of depth in 5mm-thick SS316 plate with internal defect consisting of a $1 \mathrm{~mm}$-thick layer of powder with packing fractions varying from $\eta=0.6$ to 1 .

With the most noticeable defect density confirmed, we performed another parametric study, the results of which are shown in Figure 5. This time, we gradually decreased the defect layer width from $2000 \mu \mathrm{m}$ to $10 \mu \mathrm{m}$. We maintained a packing density $\eta=0.6$ and kept the defect at $1 \mathrm{~mm}$ below the plate surface. We observed that at $1 \mathrm{~mm}$ depth, defects smaller than $50 \mu \mathrm{m}$ are not detectable with PTT. 


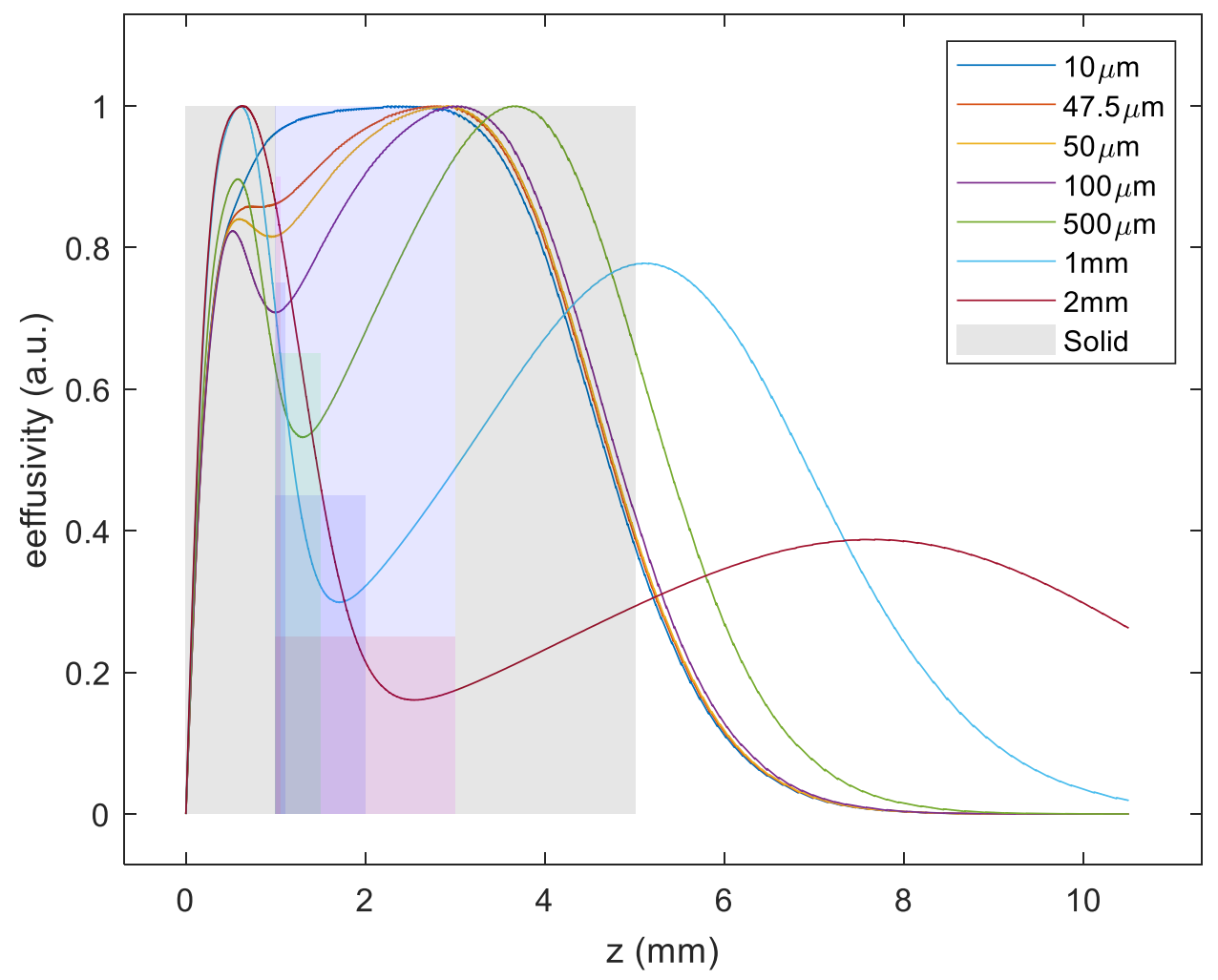

Figure 5 - Reconstructed effusivity for 5mm-thick SS316 plate with internal defects represented by powder layers with variable thickness from $2 \mathrm{~mm}$ to $10 \mu \mathrm{m}$, located at fixed depth of $1 \mathrm{~mm}$ below plate surface and fixed packing fraction of $\eta=0.6$

An analysis of Figure 5 allows us to notice a relationship between the change in effusivity $\Delta e$ and the defect's size. In fact, plotting these data points produces a logarithmic equation

$$
\Delta e=10^{0.1388} x^{0.4701},
$$

where $x$ is a non-dimensional defect size parameter, which is defined by dividing the defect layer width by the entire plate width $(5 \mathrm{~mm})$. Figure 6 contains the curve fit on both linear and logarithmic graphs. With a fit mean squared error $M S E=0.0015$, this uses a $\eta=0.6$ defect's size to predict its visibility. 

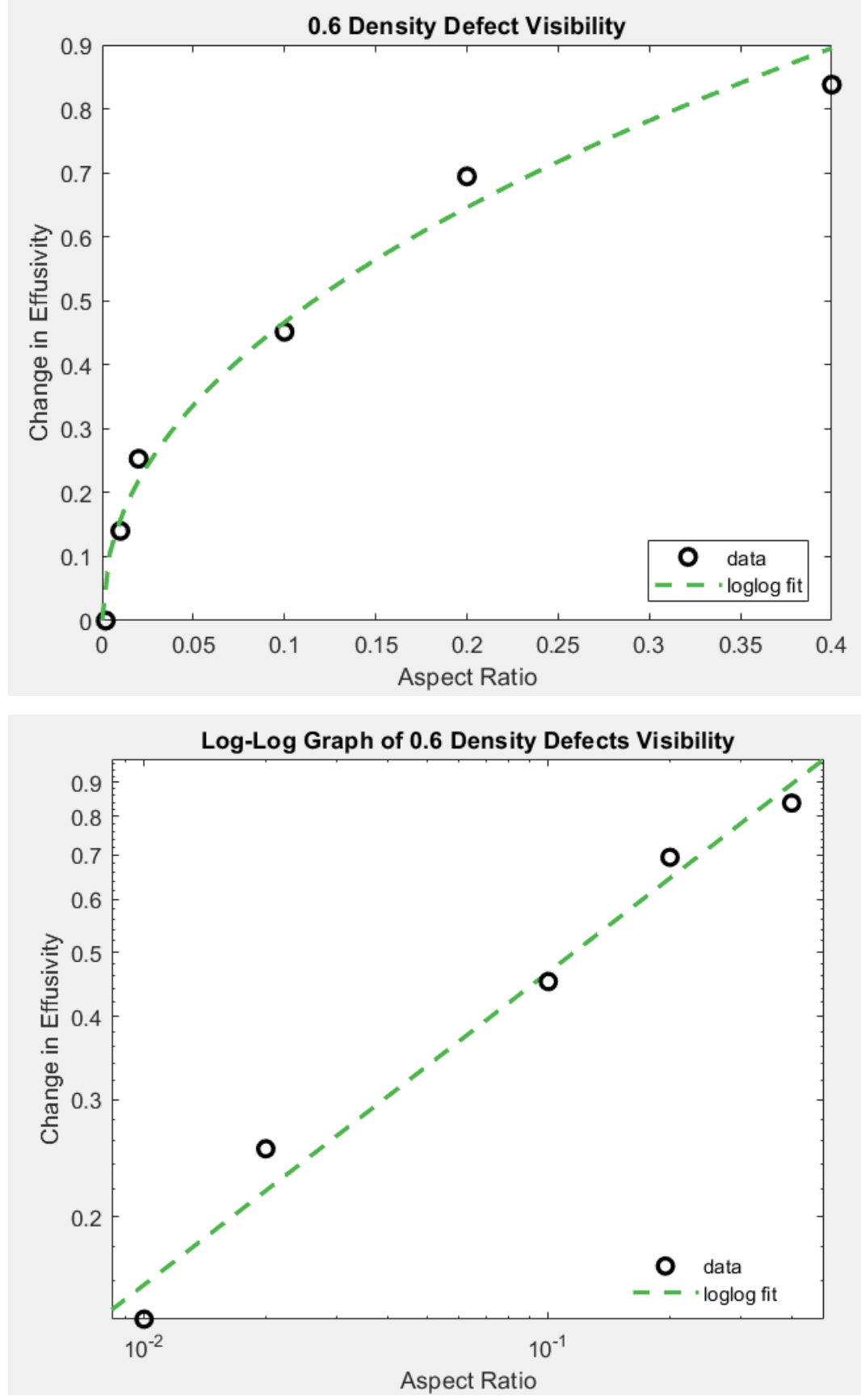

Figure 6 - The curve fit for effusivity change as a function of aspect ratio in a $\eta=0.6$ packing fraction (density) defect on linear (top) and logarithmic (bottom) scales. 


\section{Which Defects Matter?}

While developing advanced capabilities for defect detection, it is important, classify the defects which have higher potential to result in material thermal fatigue in a reactor. In principle, porosity is always expected to exist AM metallic materials simply due to manufacturing process involving rapid heating and cooling of metallic powder. Recent work reported in literature investigated critical pore size in Inconel 718 (IN718) coupons produced with selective laser melting (SLM) method [10]. Pores in the AM coupons were imaged with X-ray computed tomography (CT) at the Advanced Photon Source (APS). Critical pore size was defined as the size of a pore around which estimated stress concentrations were greater than those around other microstructural features. By applying cyclical mechanical stresses, and through crystal plasticity simulations, they determined that a pore of size smaller than $10 \mu \mathrm{m}$ was less likely to cause a crack than any other location within the structure. On the other hand, an isolated pore larger than $20 \mu \mathrm{m}$ is more likely than its surroundings to initiate a crack. While each coupon under examination was determined to have approximately 10,000 pores, only five had diameters of size $>20 \mu \mathrm{m}$. It is important to note that the study also showed that surface cracks were more prone to propagation than cracks initiated by pores. Typical environmental stresses on metallic components in nuclear reactors are temperature and corrosion. In the future, similar studies should be performed with AM specimens subjected to thermal stresses to fully understand which material defects are detrimental to the structure's function, and which defects can be disregarded. 


\section{Conclusion}

We developed COMSOL model to simulate PTT of a SS316 structure with a defect represented with a layered media model. The defect was represented as a layer consisting of un-sintered powder. Thermophysical parameters of the defect, were represented with equivalent volume model, in which powder thermal properties were scaled according to powder packing fraction. Using simulated surface temperature transients, we reconstructed the depth profile of thermal effusivity. After a series of parametric studies, we isolated packing density $\eta=0.6$ as the most visible and discovered that the smallest detectable defect at $1 \mathrm{~mm}$ depth is $50 \mu \mathrm{m}$. Given that SS316 powder grains are typically $20 \mu \mathrm{m}$ in diameter, a $50 \mu \mathrm{m}$ defect consists of approximately three un-sintered grains.

The layered media model most likely represents the upper bound of detection. Defects with finite transverse dimensions will experience lateral heat diffusion around defect boundaries, which will reduce surface temperature gradients and corresponding effusivity. The next step is to investigate detection of spheroidal-shape defects with PTT, and to investigate likelihood of crack formation due to thermal stresses. This will allow rapid qualification of AM parts for nuclear energy applications. 


\section{References}

1. A. Heifetz, X. Zhang, J. Saniie, D. Shribak, T.W. Elmer, B. Saboriendo, S. Bakhtiari, W. Cleary, "Imaging of calibrated defects in additively manufactured materials," Argonne National Laboratory, ANL-20/23 (2020).

2. S.A. Khairallah, A.T. Anderson, A. Rubenchik, W.E. King, "Laser powder-bed fusion additive manufacturing: Physics of complex melt flow and formation mechanisms of pores, spatter, and denudation zones," Acta Materialia 108, 36-45 (2016).

3. W.J. Sames, F.A. List, S. Pannala, R.R. Dehoff, \& S.S. Babu, "The metallurgy and processing science of metal additive manufacturing," International Materials Reviews, 61(5), 315-360 (2016).

4. R. Snell, S. Tammas-Williams, L. Chechik, A. Lyle, E. Hernandez-Nava, C. Boig, G. Panoutsos, and I. Todd, "Methods for rapid pore classification in metal additive manufacturing," JOM 72(1), 101-109 (2020).

5. A. Heifetz, D. Shribak, T. Liu, T. Elmer, P. Kozak, W. Cleary, "Pulsed Thermal Tomography Nondestructive Evaluation of Additively Manufactured Reactor Structural Materials," Transactions of the American Nuclear Society 121(1), 589-591 (2019).

6. A. Heifetz, D. Shribak, X. Zhang, J. Saniie, Z.L. Fisher, T. Liu, J.G. Sun, T. Elmer, S. Bakhtiari, W. Cleary, “Thermal tomography 3D imaging of additively manufactured metallic structures," AIP Advances 10, 105318 (2020).

7. H.M. Jaeger and S.R. Nagel, "Physics of granular states," Science 255(5051), 1523-1531 (1992).

8. L.C. Wei, L.E. Ehrlich, M.J. Powell-Palm, C. Montgomery, J. Beuth, J.A. Malen, "Thermal conductivity of metal powders for powder bed additive manufacturing," Additive Manufacturing 21, 201-208 (2018).

9. C.S. Kim, “Thermophysical properties of stainless steels,” ANL-75-55 (1975).

10. M. D. Sangid, P. Ravi, V. Prithivirajan, N. A. Miller, P. Kenesei and J. Park, "ICME Approach to Determining Critical Pore Size of IN718 Produced by Selective Laser Melting", JOM 72, 465-474 (2020). 


\section{Argonne}

Nuclear Science and Engineering Division

Argonne National Laboratory

9700 South Cass Avenue, Bldg. \#208

Argonne, IL 60439

www.anl.gov

Argonne National Laboratory is a U.S. Department of Energy laboratory managed by UChicago Argonne, LLC 